\title{
ROBOTS FOR CLEANING AND DECONTAMINATION OF BUILDING CONSTRUCTIONS.
}

\author{
Valery G.Gradetsky, Michael Yu.Rachkov,Sergei V.Uljanov and \\ Gorachand Nandi, Institute for the Problems in Mechanics, \\ USSR Academy of Sciences, \\ Prospect Vernadskogo 101, Moscow-117526, USSR.
}

\section{ABSTRACT}

Some important problems of building maintenance is cleaning of walls, ceilings and floors, cleaning of window glasses in high rise office buildings. The problem is specially advanced for application in nuclear power station halls, underground stations etc. Robotic systems for these purposes consist of mobile robot having horizontal motion with wall climbing robot installed on its body. Each robot is supplied with cleaning instruments. Wall climbing robot has possibility to be attached and detached with horizontal motion robot at full automatic mode. In addition to that it should be always ensured that the climbing robot at its working zone is placed in the most stable posture so that the vacuum created inside the gripper will be minimum to avoid surface damage. In this paper a method has been addressed for finding most stable posture for the robot body for a particular gripping position. Also some results of testing and experimental investigations of the floor and wall cleaning robotic system have been presented.

\section{INTRODUCTION}

The application of climbing robot(CR) is increasing day by day in the fields of different construction and maintenance operations: such as internal and external wall cleaning, ceiling and floor cleaning, window glass cleaning in high rise office building etc. The application is specially important for the periodic maintenance work inside nuclear power stations.

Design and development of vacuum leg type climbing robot has been discussed in [1] which is suitable for construction purposes. Discussions were presented for climbing type of inspection robot and climbing robot having magnetic legs in $[2,3]$.

Main principles of designing robotic complex of vertical climbing type has been discussed in [4]. Motions of CR under external disturbances have been presented in [5].

In this paper a robotic system capable of cleaning floors and walls with high degrees of mobility and reliability has been presented. In general for reliable contact on the vertical wall the CR may have different kinematic schemes, one of which has been shown in [1]. A climbing robot with vacuum paws remain attached with the vertical wall in a similar way like a lizard.

When robot starts its technological operations in any goal position the creation of vacuum to keep the robot body in equilibrium will depend on its posture. It is very important that before starting technological operations robot body must be held in most stable posture. So far a number of research work for generation of stable grasping strategies $[6,7,8,9]$ have been carried out. But all are devoted to multi fingered robot hands in manipulating type of robots. However, in this paper stable grasping strategies for CR based on two quality measures:structured twist space quality measure(Tq) and structured wrench space quality measure(Ro), have been found out. Other quality measures may also be included [10], but for simplicity and to illustrate the principle here the stable grasping strategy based on $\mathrm{T}_{q}$ and $\mathrm{R}_{q}$ have been considered. 


\section{CONSTRUCTION OF ROBOTIC SYSTEM FOR CLEANING AND DECONTAMINATION}

The whole complex of the robotic system has been shown in fig 1 , where 1 is horizontally moving robot (HMR), 2 is climbing robot, 3 \& 4 are technological equipment(vacuum cleaners)mounted on the HMR and $C R$ respectively, 5 is the manipulator for attaching and detaching the CR with HMR, 6 is the actuating devices, 7 is the controlunit, 8 is the operator, 9 is the control channel, 10 is the horizontal surface to be cleaned, 11 is the vertical surface to be cleaned.

HMR is a automatically guided vehicle for carrying manipulator for specific technological operation. HMR has the capability of moving not only on even surfaces but also on the uneven rough surfaces for the special design of wheels mounted on it. For controlling HMR in ambiguous environments the ultrasonic sensors are mounted on the body of the HMR. This proximity sensor helps the robot in navigation in cluttered environment and to find out specific objects such as doors of a room etc.

vacuum cleaners are mounted below the platform of the HMR to enable it to clean the floors on its way of movement.

During the horizontal motion the $C R$ remains idle and is being held firmly by the gripper of the manipulator mounted on HMR. When it reaches near the vertical wall the sensor gives necessary signals to the HMR controller to detach $\mathrm{CR}$ from its gripper and the manipulator attach the CR with the wall surface. After this the CR becomes functional and starts working in automatic mode for cleaning the vertical wall with vacuum cleaners attached with the CR.

The actuating device feed the CR grippers and also the vacuum cleaners through cable. The trajectory of the CR is controlled by the control unit in the programming mode.

The presence of contamination is sensed by the special sensory devices and guide the CR towards it for decontamination. After cleaning is over the $C R$ returns to its initial position and from there it is taken back to its original position by the manipulator of the HMR. The experimental result shows that the positional accuracy of detaching the CR from HMR and attaching the CR after technological operation is over and returning to its original position is $\pm 150 \mathrm{~mm}$.

The operator can monitor over the system from the video-display unit and edit the programme to change the movements of the robots if necessary. The control channel may of be wire type or wireless type. For the present complex the maximum distance between the operator and the end point of the complex is $60 \mathrm{M}$. There is a special system for winding and rewinding the cable, so that it cannot create any problem during movement.

The photographic view of the whole complex has been shown in the fig 2. Experimental results shows that the system is characterized by its reliability and mobility.

\section{MODELLING OF ORIENTATION AND MOTION OF TRANSPORT MODULE OF HMR}

Before the development of real robotic system, modelling of orientation and movement of HMR is needed to enable it to operate in a smooth and reliable manner. The main difficulty lies in the fact that the environment where the system will work is quite often undefined. To solve this problem fuzzy logic is introduced here.

It is the logic by which human being drives car. The methodology is presented in [10]. It is applicable to any complex process which is too difficult for modular control using conventional methods or the control of which is relied upon the experience of the human operator.

In this part of the article the fuzzy control of HMR with rule 
based fuzzy controller has been discussed. Control rules are derived by modelling an expert driving action. There are three methods of achieving a linguistic set of fuzzy rules which represent a systems input-output behavior:verbalization, fuzzification and identifica-

Here we have used fuzzification and identification methods. The following fuzzy control rules are used for multiple input and
single output controller [11].

$$
\begin{aligned}
& R^{i}: x_{1} \text { is } A_{1}^{i} x_{2} \text { is } A_{2}^{i} \ldots \ldots x_{n} \text { is } A_{n}^{i} \rightarrow y^{i}=p_{0}^{i}+p_{1}^{i} x_{1}+p_{2}^{i} x_{2} \\
& +p_{3}^{i} x_{3} \ldots \ldots \ldots+p_{n}^{i} x_{n} \text {. }
\end{aligned}
$$

Where $A_{j}^{2}$ s are fuzzy variables and $Y^{i}$ is the output of $i$ th The membership function of a fuzzy set lines. When the inputs $x_{i}^{\circ}-x_{n}^{\circ}$ are given, the true value of the premise of $i$ th rule is calculated as

$$
w^{i}=\min _{j=\overline{1, n}} A_{i}\left(x_{j}\right),
$$

where $A(X)$ is a membership function of fuzzy sets $A$ and the output $Y^{\prime}$ is inferred from $m$ rules by taking the weighted average of the

$$
\mathrm{Y}^{0}=\sum_{i=1}^{m} \mathrm{~W}^{i} \mathrm{Y}^{i} / \sum_{i=1}^{m} \mathrm{~W}
$$

Identification algorithms of fuz [10-13]. The reasoning methods are deszy control rules are given in in a consequence of a rule has a monotoned in $[10,12]$. A fuzzy set defuzzification is member ship function and connections are mas a weighted mean [12]. The logical The fuzzy sets are About 500 input-output data as ( $L-R$ ) type flat fuzzy numbers. ries and four variables ata have been taken from eighteen trajectofig 3 .

Here $x_{1}$ is the distance from entrance of the corner, $x_{2}$ is the distance from inner wall, $x_{3}$ is the direction (angle) of robot, $x_{4}$ is the distance from outer wall. Expert driving action are modelied in
the form of 20 control rules.

We choose three fuzzy variables for $x_{1}$, two for $x_{2}$, three for $x_{3}$ and one for $x_{4}$ by observing the expert's actions. For a given input-
output $x_{1 k}, \ldots \ldots \ldots x_{n k}$, Yk are taken from operator's control acti-
on. As in $\mathrm{Y}_{k}$ ar $\mathrm{x}_{2}$, three for $\mathrm{x}_{3}$ tions of the input space (for exampl :1) the number of fuzzy parti; small and large for $x_{2}$ etc.);2)thle small, medium and large for $x_{1}$ fuzzy variables; 3 ) the coefficients membership functions of those the number of which is $\{\operatorname{mx}(n+1)\}$. The the consequence of the rules identified using the output of i The coefficients $\left(p_{0}^{i}-p_{n}^{i}\right)$ are easily (for example as in [11] used.

Fig. 4 shows the results of the simulation of HMR on microribed in [13] programs and algorithms of simulation have been descworked very well.

\section{ANALYSIS OF STABLE GRASPING STRATEGY FOR CLIMBING ROBOTS}

Contact kinematics and transformation relations: Let us attach the following co-ordinate frames as shown in the fig 5 .
$C_{W}=$ The inertial frame, i.e., the reference frame attach 
$C_{R}=$ The body co-ordinate frame attached with the C.G. of the robot body $\mathrm{C}_{\mathrm{li}}=$ The leg co-ordinate frame attached with the last link of the leg $\mathrm{C}_{\mathrm{Li}}=$ The local co-ordinate frame attached with the $i$ th point of contact with the leg to the wall. attached at the $i$ th point of contact.

Note: The $\mathrm{Z}$ axis of $\mathrm{C}_{\mathrm{L} i}$ and $\mathrm{C}_{w i}$ coincide, $\phi$ is defined to be the contact angle relative to the two $x$ axes. This is the function of

Now it is well known that the operator $T(w)=\left[\begin{array}{ccc}0 & -w_{3} & w_{2} \\ w_{3} & 0 & -w_{1} \\ -w_{2} & w_{1} & 0\end{array}\right]$, where. $w=\left[\begin{array}{l}w_{1} \\ w_{2} \\ w_{3}\end{array}\right]$, when operated on the radius vector $\{f\}_{S}=\left[\begin{array}{l}f_{1} \\ f_{2} \\ f_{3}\end{array}\right]$ gives the following results $T(w)\{f\}_{S}=\{w x f\}_{S}=-\{f x w\}_{s}$; Using this result and transformation principle we have deduced the force/torque and velocity transformation relations between robot body to leg tips and leg tips to the leg joints. The results have been summarized below and shown in the fig 6 .

\section{Force/Torque Velocity Relations \\ Relations}

Robot body to leg tips $\left(\begin{array}{l}F_{C R} \\ M_{C R}\end{array}\right)=G X$

Leg tip to leg joints

$\tau=J_{l}^{t}(\theta) \mathrm{x}$

$$
\begin{aligned}
& \xi=G^{t}\left(\begin{array}{l}
V_{C R, C \omega} \\
W_{C R, C \omega}
\end{array}\right) \\
& \xi=J_{l}(\theta) \dot{\theta}
\end{aligned}
$$

Where $\mathrm{G}=$ Grasp matrix,

$X=T h e$ contact wrench,

$\xi=$ The contact velocity vector,

$J_{l}(\theta)=$ Leg Jacobian of the robot.

with this background we shall now try to evaluate the two quality measure $\mathrm{T}_{q}$ and $\mathrm{R}_{q}$

4.1 GRASP PLANNING There are two main steps for grasp planning 1 ) selecting a good grasp on the wall,2)using the co-operative action of the legs to take the robot body in proper grasping position.

However, in this paper we shall study how to generate the good grasp based on two quality measures; twist space quality measure and wrench space quality measure. Then the performance is being measured by constructing the performance measurement function $(P M=f(T q, R q)\}$ like

PM $=T_{q}^{\gamma} \cdot R_{q}^{1-\gamma} \ldots(4.1 .1)$, where the value of $\gamma$ will depend on the type of work the system will perform.

As for example, for stable grasping function, more weightage should be given to wrench space quality measure rather than manipulability quality which is characterized by twist space quality measure $\mathrm{T}_{q}$. We have chosen the value of $\gamma$ as 0.2 .

Our next step is to evaluate $T_{q}$ and $R_{q}$. Let us model the gripping
task by two task ellipsoids $E_{T}$ and $E_{R}$, one in twist space and ano Also let us assume stiffness control is 


$$
* \sigma_{i}=k_{i} \delta_{i} \text {, }
$$

used for the robot legs. Hence for $\operatorname{leg} \sigma_{i}$ is related to $\delta_{i}$ by*where $\mathrm{K}_{i}$ is the desired stiffness in the direction $\mathrm{D}_{i}=$ the task direction expressed in body co-ordinate.

In the generalized form we can represent

$E_{T}=\alpha E_{1} X+C(4.1 .2)$ and $E_{R}=\beta E_{2} X+d(4.1 .3)$ where $\alpha$ and $\beta$ are the scaling parameters responsible for the size of the ellipsoids and $E_{1}$ and $E_{2}$ are the structured matrix given by

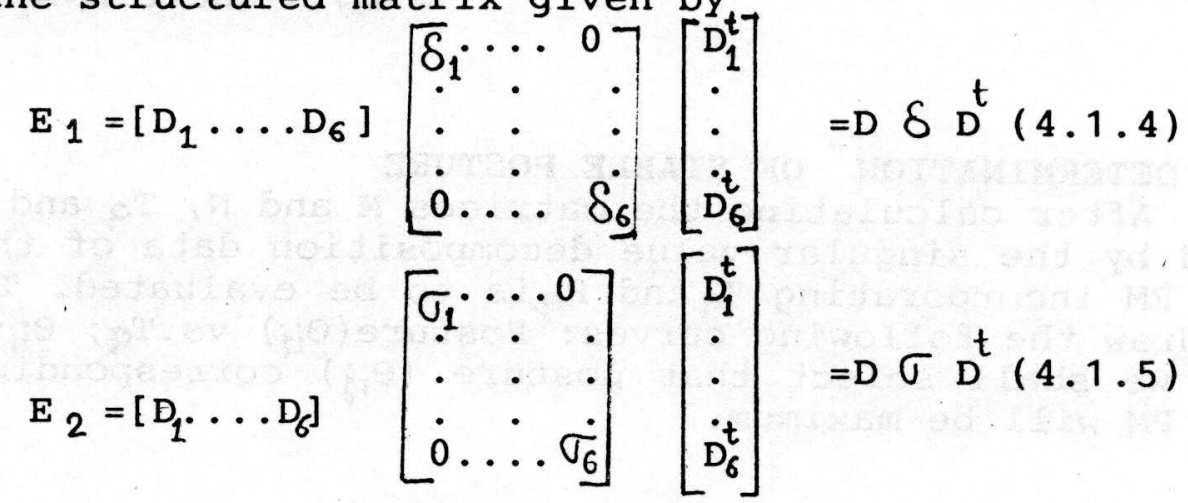

$x, c, d \in R^{6},|x| \leq 1$; for simplicity we shall assume $c=d=0$.

Structured twist space quality measure $\mathrm{Tq}$ :

Let us assume an unit ball in $R^{m}\left(O, \subset R^{m}\right)$, the space of leg joint velocities, and define the structured twist space quality $T_{q}$ by

$\mathrm{T}_{q}=\operatorname{Sup}_{\alpha \in R}\left\{\propto\right.$, such that $\left.J_{l}\left(O_{1}^{m}\right) \supset \mathrm{G}^{t}\left(\mathrm{E}_{T}\right)\right\}$

$q \alpha \in R_{\text {The }}$ physical meaning of $\mathrm{Tq}$ is as follows: The unit ball $\mathrm{O}_{1}^{\text {min }}$ the leg joint velocity space is mapped into the space of contact veloci$t_{y}$ by $J_{l}$. On the other hand, gripping task ellipsoid is mapped back into the contact velocity space by $G$. Tqis then the largest $\propto$ such that $G\left(E_{U}\right)$ is contained in $J_{l}\left(O_{1}\right)$ (ref. fig.7). In other words the robot body velocity of size $\propto$ can be accommodated by leg joint velocity of unit magnitude. Theoretically Tqis the ratio of structured output (the task ellipsoid)over the input(i.e. the leg joint velocity).

From fig.7, it is evident that $\mathrm{T}_{\mathrm{q}}$ is at its maximum if the inner ellipsoid has the same size and orientation as the outer ellipsoid.

Now using the expression $J_{l}\left(O_{1}^{m}\right)=\alpha E_{1} X \in R_{1}^{n}\left\langle\alpha E_{1} X, \quad\left(J_{l} J_{l}^{t}\right)^{-1} \alpha E_{1} X\right\rangle \leqslant 1$ and $\underset{G(E)}{t}=\left\{\alpha G^{t} E_{1} x \in R^{n}: x \in R^{6} \quad|x| \leq 1\right\}$

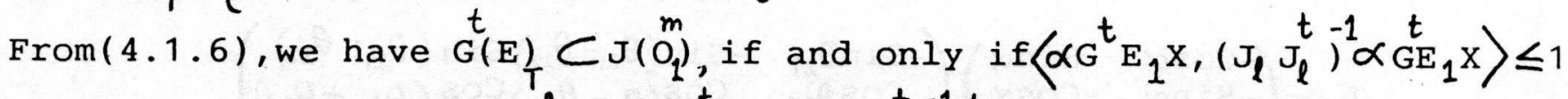
for $\operatorname{all}|x| \leqslant 1, \quad$ or, $\alpha^{2} \operatorname{Sup}\left\langle G^{t} E_{1} x,\left(J_{l} J_{l}^{t}\right)^{-1} G^{t} E_{1} x\right\rangle \leqslant 1$

$$
\text { or, } \alpha^{2} \sup \left\langle x,\left(G^{t} E_{1}^{t}\left(J_{l} J_{l}^{t}\right)^{-1} G^{t} E_{1} x\right\rangle \leq 1\right. \text {, which is }
$$

equivalent to $\alpha \leq \sigma_{\max }^{-1 / 2}\left\{E_{1}^{t} G\left(J_{l} J_{l}^{t}\right)^{-1} G^{t} E_{1}\right\}$.

or $\alpha \leq \sigma_{\max }^{-1 / 2}$ where $M=\left\{E_{1}^{t} G\left(J_{l} J_{l}^{t}\right)^{-1} G^{t} E_{1}\right\}$ and $\sigma_{\max } M$ is the maximum singular value of the matrix M. Hence $\operatorname{from}(4.1 .6), \quad \mathrm{T} q=\sigma_{\max }^{-1 / 2} \mathrm{M}$. (4.1.8.a)

Structured wrench space quality measure $\mathrm{R}_{q}$ : To evaluate wrench space quality $R q$ let $O_{1}^{n} \subset R^{n}$ be the unit ball in the leg wrench space and $\sigma_{m}\left(J_{l}\right)$ be the maximum singular value of $J_{l}$. We define the structured wrench space quality 


$$
R_{q}=\operatorname{Sup}_{\beta \in R}\left(\beta \text {, such that } G\left(O_{1}^{n}\right) \supset E_{R}\right) \cdot \sigma_{\text {max }}^{-1}\left(J_{l}\right)(4.1 .9)
$$

The physical meaning of $R_{q}$ has been elaborated in the fig.8. As before it can be evaluated as

$$
\begin{aligned}
\mathrm{R}_{q} & =\sigma_{\max }^{-1 / 2} \cdot\left\{\mathrm{E}_{2}^{t}\left(\mathrm{G} \mathrm{G}^{-1} \mathrm{E}_{2}\right) \cdot \sigma_{\max }^{-1}\left(J_{l}\right)\right\} \\
& =\sigma_{\max .}^{-1 / 2} \mathrm{~N} \text { where } \mathrm{N}=\mathrm{E}_{2}^{t}\left(\mathrm{G} \mathrm{G}^{t} \mathrm{E}_{2} \cdot \sigma_{\max }^{-1}\left(J_{l}\right)\right. \\
& (4.1 .8 \cdot \mathrm{b})
\end{aligned}
$$

\subsection{DETERMINATION OF STABLE POSTURE}

After calculating the matrices $M$ and $N, T_{q}$ and $R_{q}$ can be evaluated by the singular value decomposition data of the matrices. Then the $\mathrm{PM}$ incorporating $\mathrm{T}_{q}$ and $\mathrm{R}_{\mathrm{q}}$ is to be evaluated. Then we will have to draw the following curves: Posture $\left(\theta_{i j}\right)$ vs.Tqi $\theta_{i j} v s . R q$ and $\theta_{i j}$ vs.PM, and we shall select that posture $\left(\theta_{i j}\right)$ corresponding to what $T_{q}, R_{q}$ and PM will be maximum.

4.3 EXAMPLE AND SIMULATION For simplicity let us consider a two dimensional model. (ref. marked part of fig. 5.). Let 1) the contact be modelled as point contact with friction, 2) the leg spacing be of 2 unit, 3) G is fixed as shown in the fig.5,4) the robot body is constrained to move vertically. This leaves the system with a single degree of freedom. Let $\theta_{i j}$ be the generalized co-ordinate of the system and we study how $\theta_{i j}$ affects the structured grasp quality measure.

Construction of grasp matrix G:

Steps which are to be followed for constructing the grasp matrix are 1) specify a body co-ordinate and obtain the co -ordinates of each contacting point;2) determine the unit normal and two orthogonal tangent vectors to the contacting surface at the contact point; 3)pick up a torque origin in body co ordinates and construct for each contact map the contact matrix;4) join the contact matrices side by side into a big matrix. This is the grasp matrix for the particular choice of the body co-ordinates and the particular choice of the torque origin.

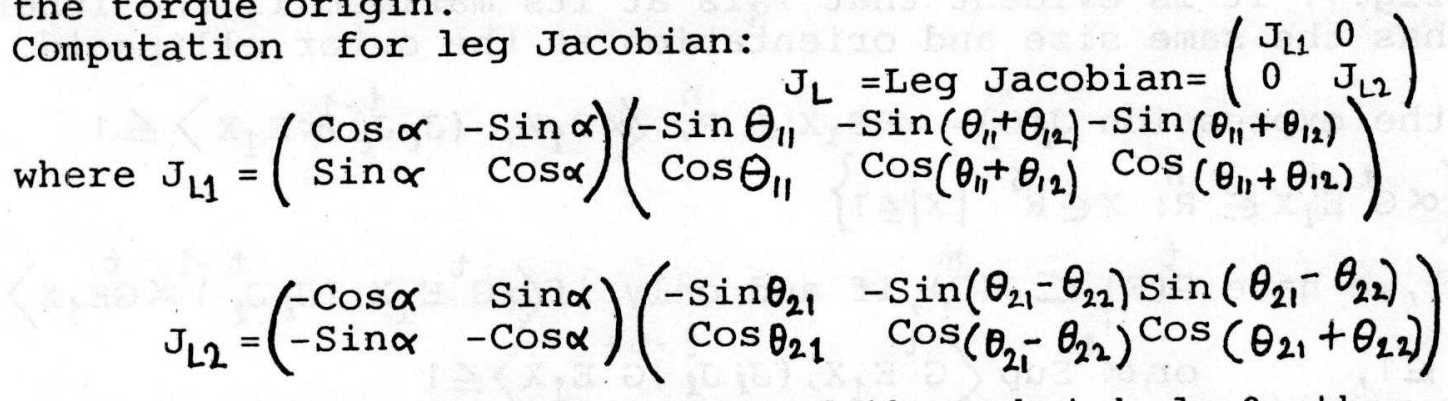

where $\alpha=$ the orientation angle of the robot body $=0$; other constrains are $\theta_{12}=\pi-2 \theta_{11}, \theta_{21}=\pi-O_{11}$, and $O_{11}-O_{22}=\pi \quad-\left(\theta_{11}+\theta_{12}\right)$

Other computations : value of $K=\left(\begin{array}{ccc}10 & 0 & 0 \\ 0 & 190 & 0 \\ 0 & 0 & 100\end{array}\right) \quad D=I$, Unit matrix.

$\delta=\operatorname{diag}\left\{\begin{array}{lll}0.8 & 0.7 & 0.02\end{array}\right\} ;$
$\sigma=\operatorname{diag}\left\{\begin{array}{lll}8 & 133 & 2 .\end{array}\right\}$.

With reference to the marked part of fig.5, for two contact points the grasp matrix $G=\left(\begin{array}{rrrr}-1 & 0 & 1 & 0 \\ 0 & -1 & 0 & 1 \\ 0 & -1 & 0 & -1\end{array}\right)$

Knowing $\mathrm{D}, \delta \& \sigma$, structured matrices $\mathrm{E}_{1} \& \mathrm{E}_{2}$ can be computed 
from (4.1.4) \& (4.1.5), hence we can calculate $\mathrm{Tq} \&$ Rq from (4.1.8.a) \& (4.1.8.b) respectively by singular value decomposition data of $M$ and $N$. Fig.9 shows plots of quality measure and the performance measure as a function of $\theta_{11}$. It is evident from this figure that the optimal posture of $C R$ is corresponding to the position $A$ where $\theta_{\|}=0.7$ radian $=40$ degrees approximately.

\section{CONCLUSIONS}

The mobile complex including horizontal moving robot and climbing robot has got tremendous practical applications in the fields of building construction. As a future work, for reliable functioning of such complex in real environment, several sensory devices together with the development of mathematical models for different variants of the system and the elements of artificial intelligence are to be incorporated.This will give the possibility to control the robotic complex with high accuracy, to test the quality of the technological operation and to enable the robotic system to work in an undefined environment.

\section{REFERENCES}

1) A.A.Collie,J.Billingsley,E.Vonputtkamer-"Design and performanceof the portsmouth climbing robot"-Proc.7th ISARC,5-7 June1990 Bristol, England.

2) Sujiyata S, Naiton S, Sato C,Ozaki N,Watahikis, 1986, "Wall surface vehicle with magnetic legs or vaccuum legs".Proc.of the 16th ISIR Brussels, Belgium, pp691-696.

3) Fujita A,Tsnge M, Morik, Sonoda S, Watahikis, 1986. "Development of inspection robot for spherical gas storage tanks", proc.of the 16 th ISIR , Brussels, Belgium, pp1185-1194.

4) Gradetsky V.G, Rachkov M.Yu. "Wall climbing robot and its applications for building construction",PrOc.7th ISARC,5-7 June 1990, Bristol, England.

5) Gradetsky, V.G,Akselrod B.V, 1989 " Motion of wall surface robot under external disturbances"-proc.of the 20th ISIR, Tokyo, Japan.

6) Zexiang Li,Ping Hsu, Shankar Sastry " Grasping and co-ordinated Manipulation by a multifingered robot hand"-Int.J. of Robotics Research.Vol.8. No4, 1989.

7) Van-Duc Nguyen, "Constructing Stable Grasp" Int.J.of Robotics Research.Vol.8.No1, 1989.

8) Salisbury J.K."Articulated Hands:Force control and kinematic issues"Proc.of Joint Automatic Control Conference,Virginia, June 1981 .

9) Zexiang Li,S.Sankar Sastry."Task oriented optimal grasping by Multifingered Robot Hands" IEEE J.of Robotics and Automation Vol. 4, No.1, 1988 .

$10)$ Petrov B.N, Ulanov G.M, Uljanov S.V,"Theory of Modelling in controlling the processes"(in Russian)-Moscow, Nauka, 1978.

11 ) Sugeno M,Nishida M, "Fuzzy control of model car" Fuzzy sets and systems-1985, Vol.16, No.2, page 103-113.

12 ) Sugeno M, Murofushi T et.al."Fuzzy algorithmic control of a model car by oral instructions", Fuzzy sets and systems-1989, Vol.32, No. 2, page 207-219.

13) Gradetsky V.G, Rachkov M.U,Sizov U.G,"System Modelling of vertical climbing Robots" (in Russian), Ijvestia, AN SSSR, Technichiskaya Kibernetika, No.4,1991. 


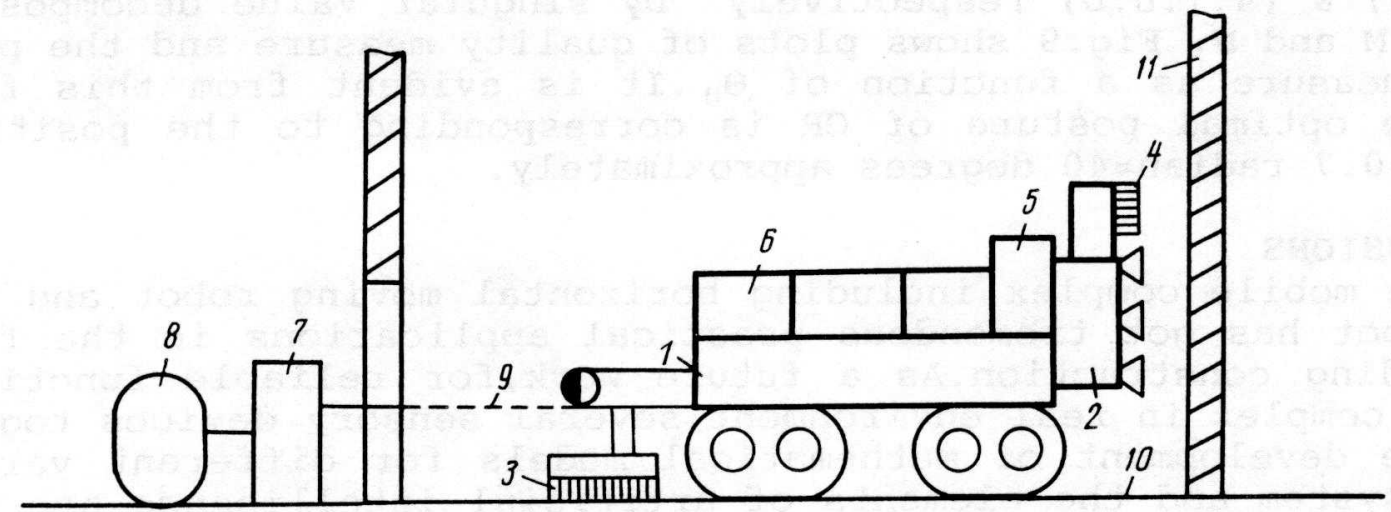

Fig. I Robotic system complex。

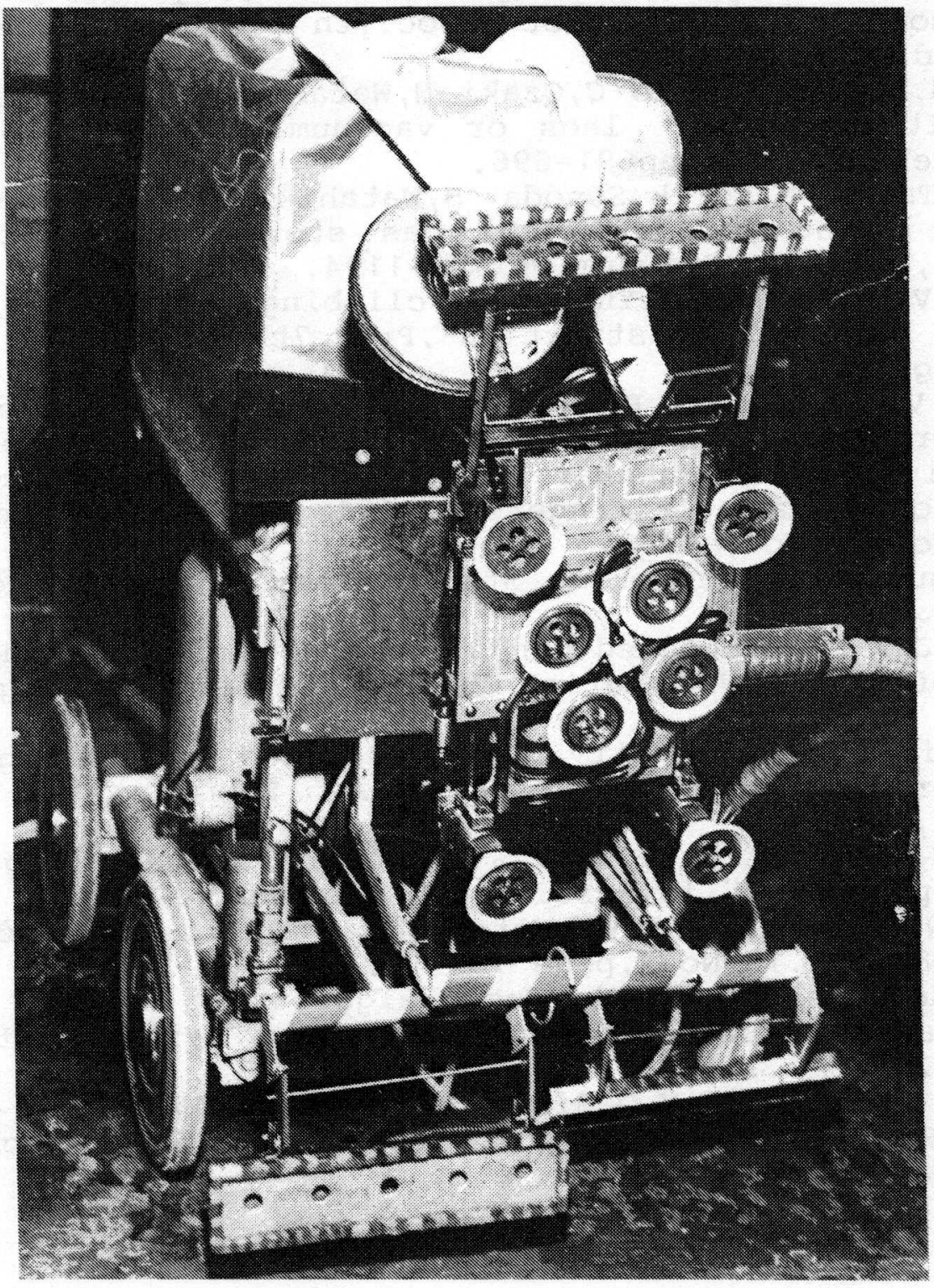

Fign2 Photogrambic view of the robotic complex. 


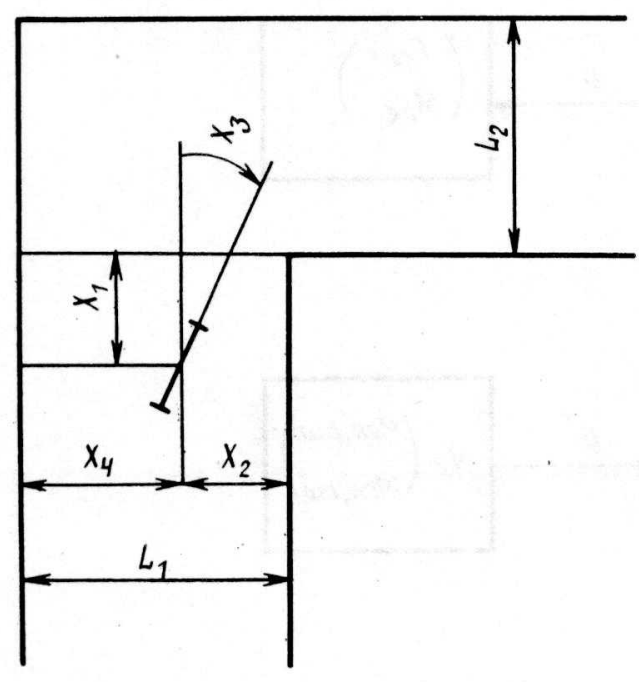

Fig. 3

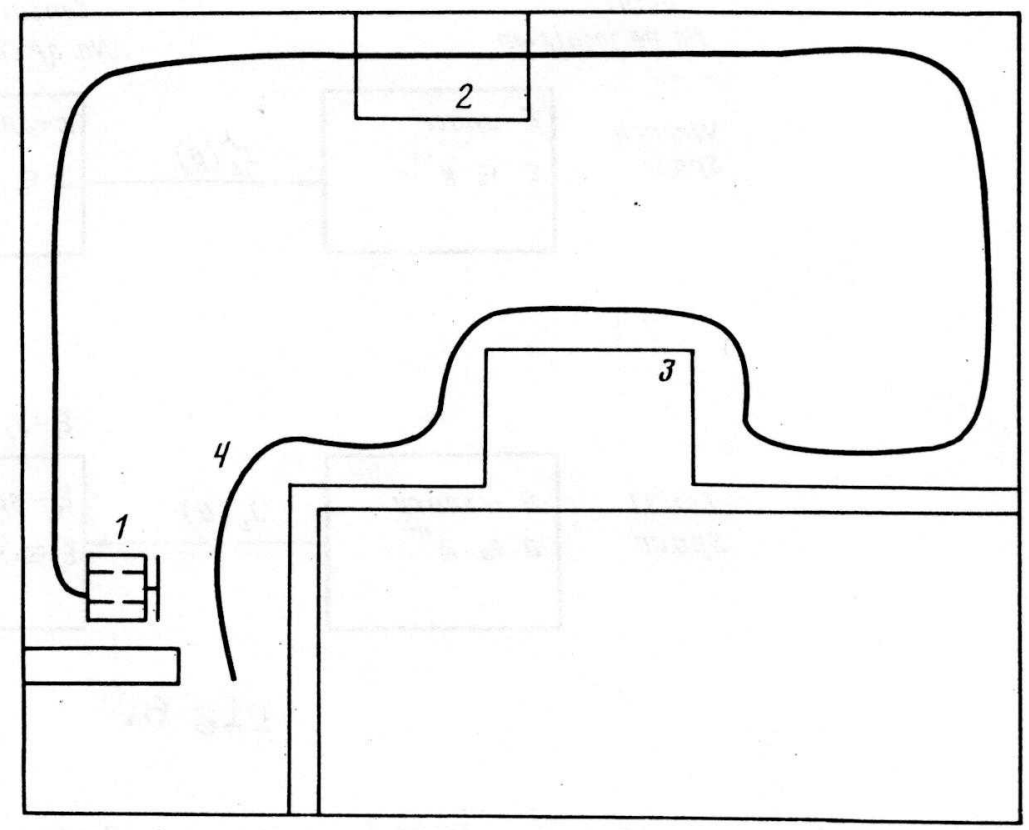

Fig. 4 Results of simulation of HMR, I- HMR,2- surface to be cleaned, 3- obstacle, 4-Trajectory of HMR.

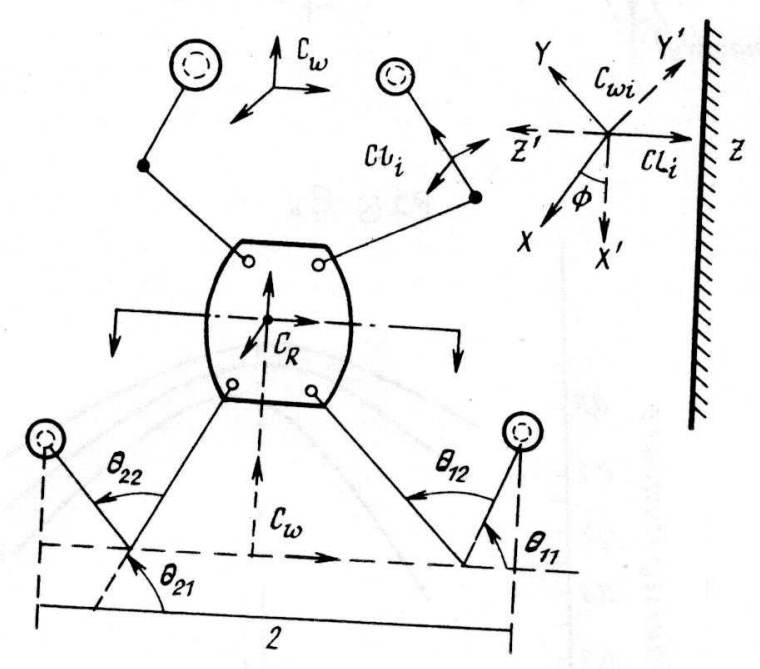

Fig. 5 Co-ordinate system of climbing robot. 

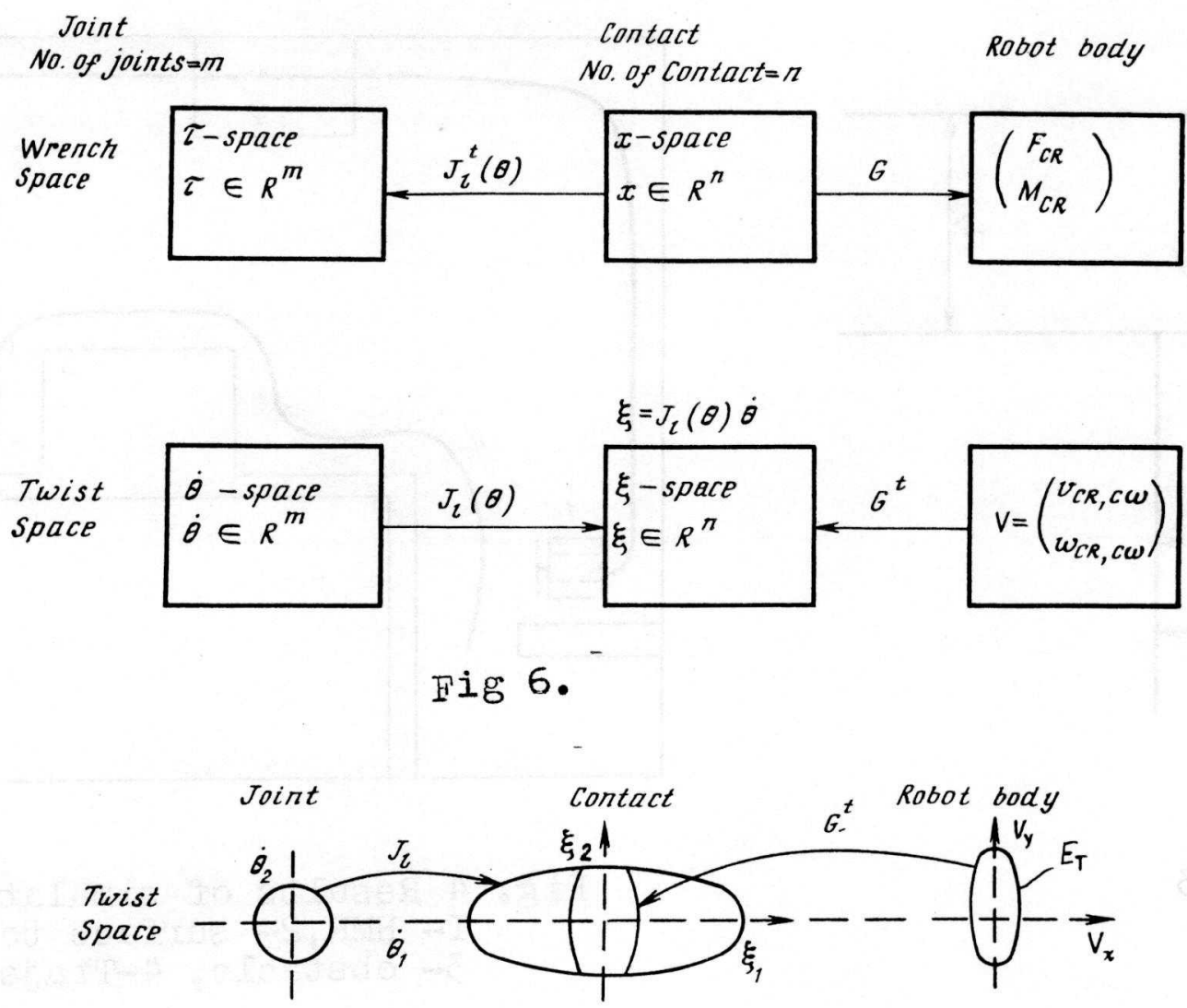

Fig 7.

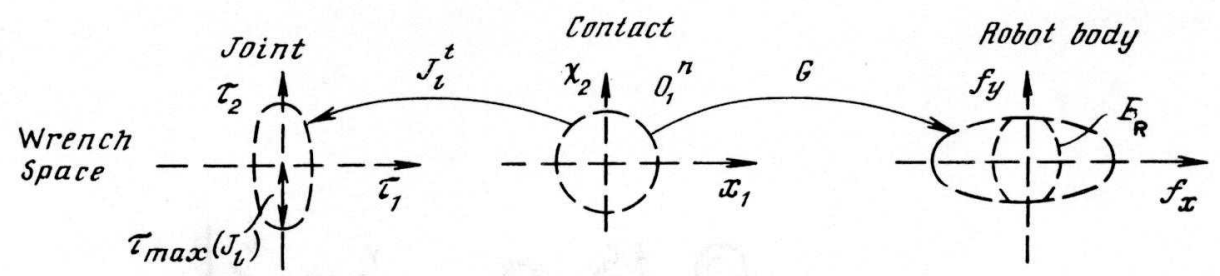

Fig 8.

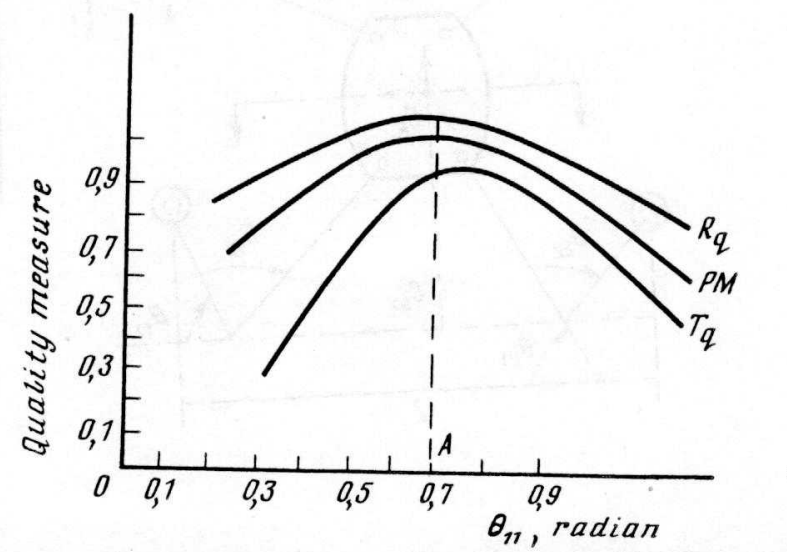

Fig 9. 